\title{
Fabrication and evaluation of GaN negative and bifocal microlenses
}

\author{
H. W. Choi, ${ }^{a}$ E. Gu, C. Liu, J. M. Girkin, and M. D. Dawson \\ Institute of Photonics, University of Strathclyde Wolfson Centre, \\ 106 Rottenrow, Glasgow G4 ONW, United Kingdom
}

(Received 2 November 2004; accepted 17 December 2004; published online 11 March 2005)

\begin{abstract}
Methods of fabricating negative and bifocal microlens arrays have been demonstrated in this paper. The technique of photoresist molding using a sapphire positive lens template was used for the patterning of negative microlenses, while the bifocal microlens arrays were fabricated using a two-step etch process. In both cases, the lenses were etched using inductively coupled plasma. Microlenses with diameters as small as $10 \mu \mathrm{m}$ have been demonstrated and were characterized using atomic force microscopy and confocal microscopy. The lens arrays were found to be smooth, uniform, and to have focal lengths consistent with their design and calculated values. (C) 2005 American Institute of Physics. [DOI: 10.1063/1.1857062]
\end{abstract}

\section{INTRODUCTION}

Individual microlenses, or arrays thereof, have a diverse range of applications in optical systems. These applications can be grouped into three generic areas: (1) beam shaping, (2) interconnections, and (3) imaging. ${ }^{1}$ While there are numerous approaches toward microlens fabrication, the method of melting photoresist is the most commonly used. However, these techniques are only suitable for the fabrication of single-power positive lenses. ${ }^{2}$ While the focusing and collimation abilities of positive lenses are suitable for many applications, there are instances where flexibility in the lens shape is desirable.

In this paper, we present approaches to the fabrication of bifocal and negative microlens arrays, respectively, using alternative photoresist-shaping techniques. Bifocal microlenses, which have the capability of producing two focal points simultaneously along the optical axis, have potential applications, for example, in focusing laser light onto two separate planes on dual-layer disks or for multiple-layer optical tweezer configurations. Negative microlenses, on the other hand, can produce divergent beams from a collimated source and their use in combination with the more conventional positive lenses enables complete micro-optical systems to be designed and fabricated.

The fabrication of negative microlenses by laser micromachining has been reported recently, although a number of problems with the technique have been identified, including limitations on optical element dimensions, challenges in array scaling, and poor surface quality (high roughness). ${ }^{3}$ This paper describes the method of making negative microlenses by photoresist molding. Bifocal microlenses are produced using a combination of resist reflow and controlled two-stage plasma etching. In both formats of microlens arrays, the individual lenses fabricated have diameters ranging from 10 to $100 \mu \mathrm{m}$.

\footnotetext{
a) Author to whom correspondence should be addressed; present address: Department of Electrical and Electronic Engineering, University of Hong Kong, Pokfulam Road, Hong Kong; electronic mail: hwchoi@eee.hku.hk
}

\section{EXPERIMENTAL DETAILS}

The microlens arrays in this work were fabricated on GaN epitaxial layers, although the approaches taken are also applicable to other hard optical materials including silicon carbide, sapphire, and diamond. Positive microlens arrays in $\mathrm{GaN}$ and GaN-based devices have been reported recently in detail by Oder $e t a l .{ }^{4}$ and Choi et $a l .{ }^{5}$ respectively, where the importance of being able to form micro-optical elements ${ }^{6}$ from this technologically significant material was highlighted. Here we show that negative and bifocal lenses can also be fabricated from epitaxial GaN. A 3.4- $\mu$ m-thick undoped GaN epi film was grown on $c$-plane sapphire by metal organic chemical vapor deposition (MOCVD) under standard conditions. Samples of $10 \times 10 \mathrm{~mm}^{2}$ size were cleaved from the wafer, and a 7- $\mu \mathrm{m}$-thick layer of photoresist (Shipley SPR220-7.0) was spin coated onto the GaN surface of each. The lenses were subsequently shaped using the methods described in the following sections. The resulting patterns in the photoresist layer were transfered into the GaN by inductively coupled plasma (ICP) etching in an STS Multiplex ICP system. Details of the etching process have been reported previously. ${ }^{7}$ The resulting microlenses were characterized by atomic force microscopy (AFM) for the assessment of the profile and surface roughness. The optical properties of the lenses were evaluated by reflection-transmission confocal microscopy, ${ }^{8}$ by which the focal lengths were accurately measured.

\section{NEGATIVE MICROLENSES}

The photoresist "dimples" for fabricating the arrays of negative lenses were formed by placing a mold onto the photoresist surface. The mold used in this work was a positive microlens array fabricated on sapphire, with microlens elements having diameters ranging from 10 to $100 \mu \mathrm{m}$, a center height of $1.9 \mu \mathrm{m}$, and a surface roughness of less than $3 \mathrm{~nm}$. A $20-\mathrm{nm} \mathrm{SiO}_{2}$ layer was deposited onto the surface of the positive lens array to act as a sacrificial lift-off layer [Fig. 1(a)]. The sapphire microlens template was then pressed against the photoresist layer on the GaN substrates and the assembly placed on a hot plate, set to $120^{\circ} \mathrm{C}$, to enable 


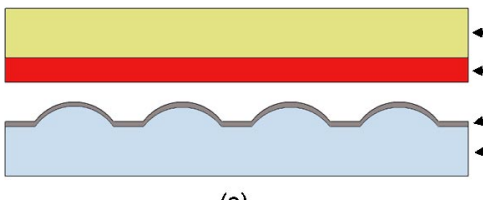

(a)

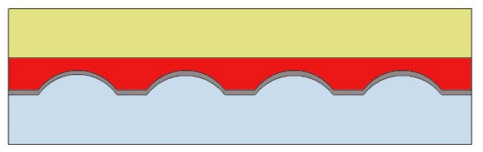

(b)

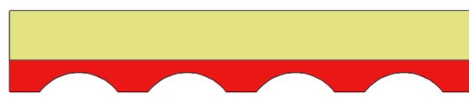

(c)

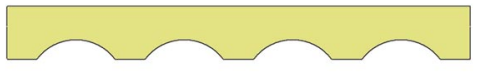

(d)
- substrate

—photoresist -silica coating -microlens template

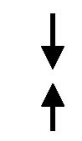

separation by lift-off in $\mathrm{HF}$ negative lens transferred onto substrate by dry etching
FIG. 1. (Color online) Process flow for the fabrication of negative microlenses. (a) A photoresist layer was spin coated onto a $\mathrm{GaN}$ substrate, while a 20- $\mu$ m-layer of $\mathrm{SiO}_{2}$ was deposited onto the sapphire microlens template. (b) The template and substrate are pressed and the negative lens pattern is transferred onto GaN by ICP etching.

resist melting and reflow. Force was applied by pressing a 2-kg weight onto the substrate in order to remove trapped air in the photoresist layer [Fig. 1(b)]. The assembly was subsequently cooled (using a stream of $\mathrm{N}_{2}$ ) and immersed into a 7:1 buffered oxide etch (BOE) solution [Fig. 1(c)]. The sacrificial $\mathrm{SiO}_{2}$ layer was then gradually etched away, resulting in the separation of the sapphire lens template and the GaN substrate, and a negative microlens pattern being left in the photoresist layer. This pattern was then transferred into the $\mathrm{GaN}$ material by ICP etching using $\mathrm{Cl}_{2} / \mathrm{Ar}$.

Figure 2(a) shows an AFM image of an individual negative microlens with a diameter of $35 \mu \mathrm{m}$. From the crosssectional profile, the center height (depth) was determined to be $1.1 \mu \mathrm{m}$ with a surface rms roughness of $\sim 3 \mathrm{~nm}$ over a $3-\mu \mathrm{m} \times 3-\mu \mathrm{m}$ area. Using these parameters, the focal length of the lens was calculated to be $-112 \mu \mathrm{m}$, using the method outlined in Ref. 6. The cross-sectional profile of the lens, extracted from the AFM scan, is plotted in Fig. 2(b). The experimentally obtained profile was then compared to the theoretical curvature of a circle using a chi-squared fitting algorithm, which is also plotted in Fig. 2(b). It can be seen that the maximum deviation from the theoretical profile is about $20 \mathrm{~nm}$.

Figure 3 shows the cross-sectional ( $X-Z$ plane) confocal microscope image of a GaN negative microlens array of lens diameter $35 \mu \mathrm{m}$. The confocal technique allows the direct imaging of successive planes of incident, emitted, or transmitted light through (from) a microscale optical element. We follow the approach we demonstrated in Ref. 8, utilizing a collimated 532-nm Nd: yttrium aluminum garnet (YAG) laser for illumination, in a confocal system allowing beam sectioning with axial and lateral resolutions of $0.8( \pm 0.1) \mu \mathrm{m}$ and $0.25( \pm 0.02) \mu \mathrm{m}$, respectively. It can clearly be seen that collimated light rays diverge after they pass through the lenses, showing the desired functionality of the negative lens. By extending traces of the light rays passing through the lens back to a virtual focal point behind the lens as

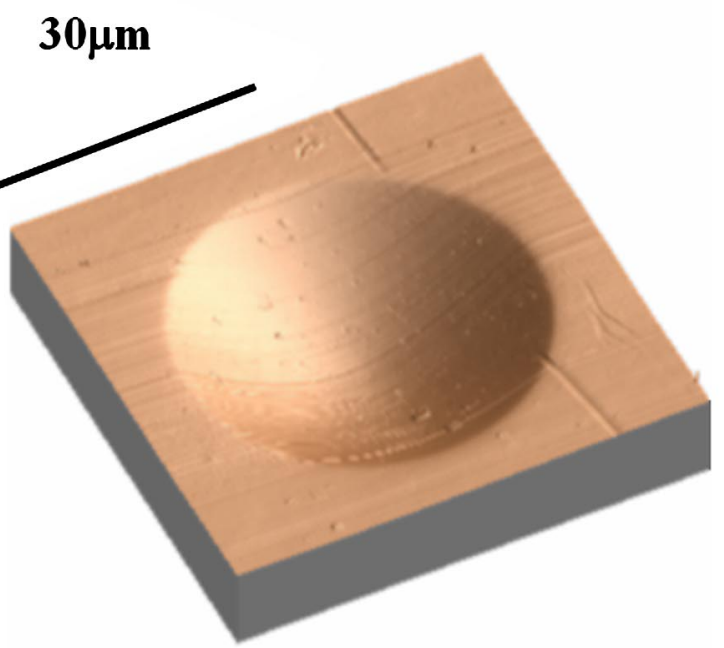

(a)

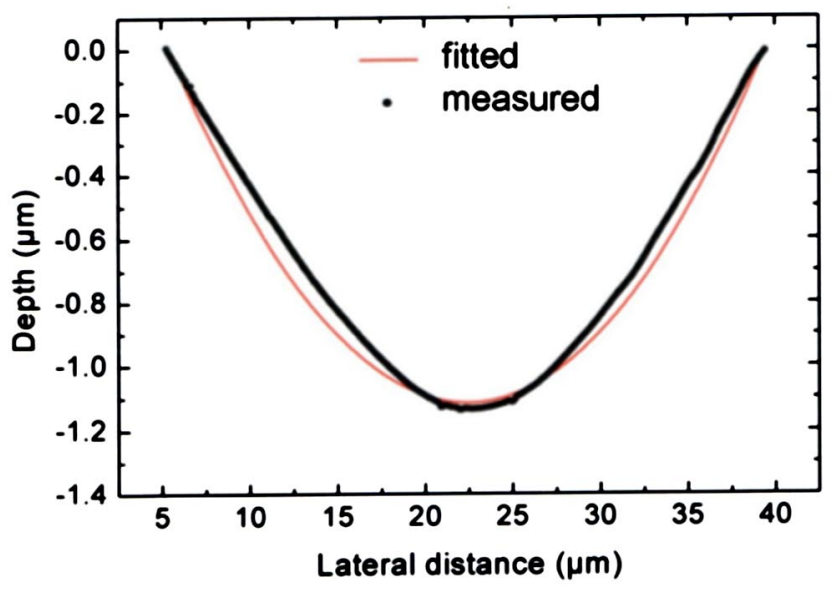

(b)

FIG. 2. (Color online) (a) AFM 3D image showing a fabricated negative microlens. The cross-sectional profile of the lens, together with the fitted curve, is shown in (b).

shown in Fig. 3, the focal length of these negative lenses can be determined. From this image, the focal length of these negative lenses is measured to be $-125 \mu \mathrm{m} \pm 2 \mu \mathrm{m}$, which is in reasonable agreement with the calculated value.

\section{BIFOCAL MICROLENSES}

The initial processing sequences in the fabrication of bifocal microlenses are identical to those of conventional single, positive-curvature lenses. ${ }^{2}$ The photoresist layer was patterned by photolithography to form arrays of disks with a range of diameters from 10 to $100 \mu \mathrm{m}$. The disks were subsequently melted in an oven, where the temperature was ramped to $150{ }^{\circ} \mathrm{C}$ in three successive stages, forming spherical caps as a result of resist reflow.

To achieve microlenses with dual curvatures, a two-stage etching approach was adopted. Since the curvature of an etched lens is primarily determined by the etch selectivity of the etch process, two separate etch recipes have been devel- 


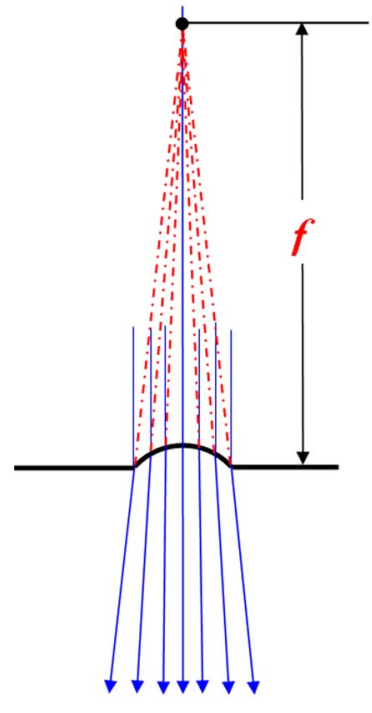

(a)

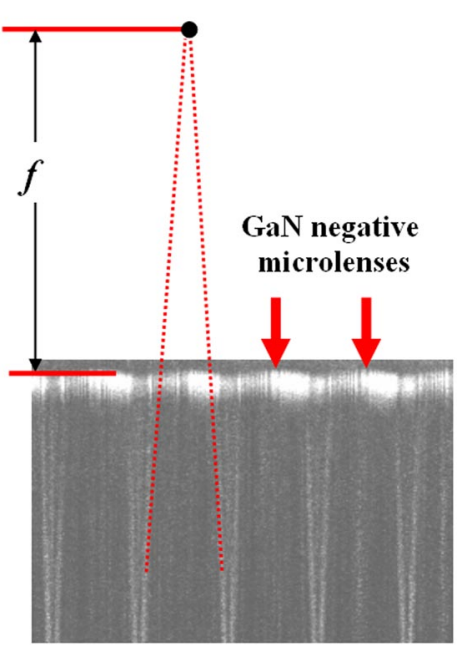

(b)
FIG. 3. (Color online) (a) Schematic diagram depicting the light rays through a negative lens. The actual light pathways through the GaN negative microlenses are taken using a confocal microscope, as shown in (b). The focal length was determined by extrapolating the light rays to an external focal point.

oped. The lower, or base, lens was etched using a $\mathrm{Cl}_{2} / \mathrm{BCl}_{3}$-based plasma, at coil and plate powers of 300 and $150 \mathrm{~W}$, respectively (giving an etch selectivity between photoresist and $\mathrm{GaN}$ of 1 to 1 ), while the upper, or central, lens was etched in a $\mathrm{Cl}_{2}$ /Ar-based plasma, at coil and plate powers of 400 and $300 \mathrm{~W}$, respectively (giving an etch selectivity of 1.7 to 1 ). The difference in selectivity between the two-etch process enabled the fabrication of the dual-power microlens. A reflection image taken under a conventional microscope focused at the substrate surface of a bifocal GaN microlens, fabricated as above, is shown in Fig. 4.

A cross-sectional ( $X-Z$ plane) confocal image of a GaNbifocal microlens array is shown in Fig. 5. For these bifocal lenses, the lower element has a diameter of $66 \mu \mathrm{m}$ and the diameter of the top element is $42 \mu \mathrm{m}$, as shown in Fig. 4. It

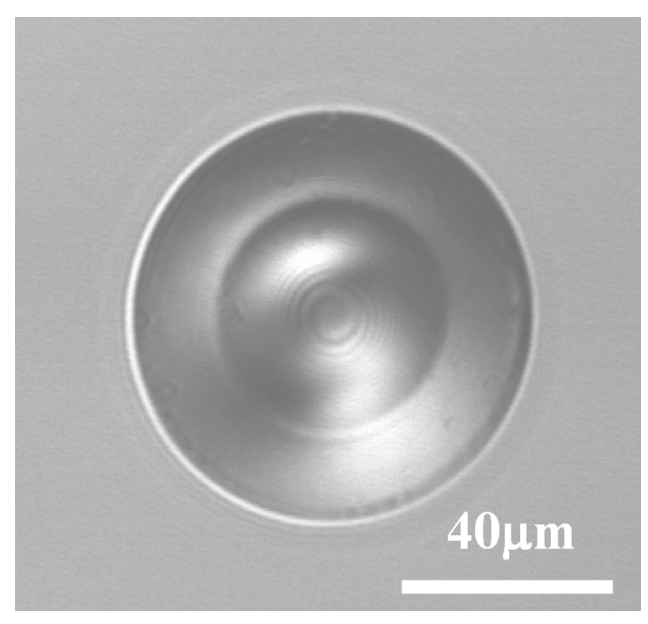

FIG. 4. (Color online) Reflection image focused at the substrate surface of a GaN bifocal microlens.

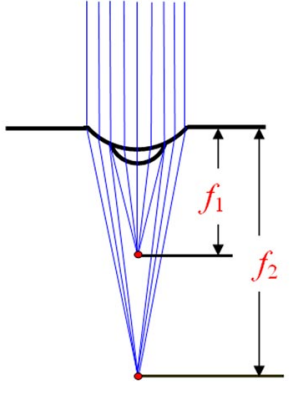

(a)

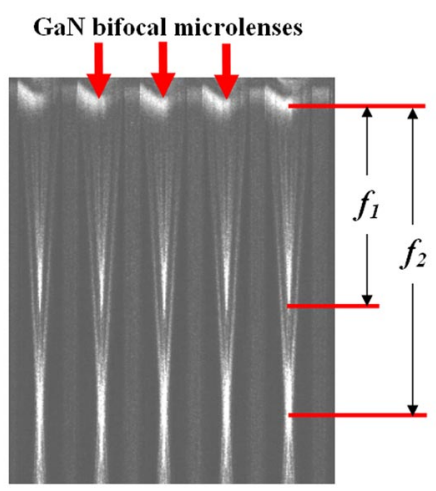

(b)
FIG. 5. (Color online) (a) Schematic diagram showing the light pathways through a bifocal microlens. Confocal microscope images showing the focusing properties of bifocal microlenses are illustrated in (b). Two distinctive focal points can be identified.

is evident that collimated light rays, after they pass through the lenses, converge at two distinct focal points. From this image, the two focal lengths of the bifocal lens are measured to be $f_{1}=74 \pm 2 \mu \mathrm{m}$ and $f_{2}=124 \pm 2 \mu \mathrm{m}$, respectively. These measured focal lengths are again close to the calculated values of $78 \pm 2 \mu \mathrm{m}$ and $130 \pm 2 \mu \mathrm{m}$, based on the dimensions recorded from the AFM data, which also indicated a highquality surface finish to the lenses. This image also shows that all the lenses measured have the same focal length, demonstrating high uniformity of the microlens array.

\section{CONCLUSION}

In summary, negative and bifocal microlenses have been fabricated on $\mathrm{GaN}$ using different processing techniques. The negative microlenses were fabricated using a photoresist molding technique, whereas the bifocal lenses were formed in two steps using etch recipes utilizing different etch selectivites. The microlenses have diameters ranging from 10 to $100 \mu \mathrm{m}$, with surface roughness better than $3 \mathrm{~nm}$. The focal lengths of the lenses, as determined by confocal microscopy, were in line with the values calculated using dimensions based upon AFM measurements. These techniques facilitate complementary optical elements to the more standard positive spherical microlenses, and open up true microoptical systems applications of microlenses. In addition, the techniques demonstrated here are applicable to other technologically important hard optical materials, including diamond, sapphire, and silicon carbide.

${ }^{1}$ D. Daly, Microlens Arrays (Taylor \& Francis, New York, 2001).

${ }^{2}$ H. W. Choi, E. Gu, C. Liu, C. Griffin, J. M. Girkin, I. M. Watson, and M.

D. Dawson, J. Vac. Sci. Technol. B 23, 130 (2005).

${ }^{3}$ K. L. Boehlen and I. S. Boehlen, Proc. SPIE 5339, 118 (2004).

${ }^{4}$ T. N. Oder, J. Shakya, J. Y. Lin, and H. X. Jiang, Appl. Phys. Lett. 82, 3692 (2003).

${ }^{5}$ H. W. Choi, C. Liu, E. Gu, G. McConnell, J. M. Girkin, I. M. Watson, and M. D. Dawson, Appl. Phys. Lett. 84, 2253 (2004).

${ }^{6}$ H. W. Choi, C. W. Jeon, M. D. Martin, P. R. Edwards, R. W. Martin, and S. Tripathy, J. Appl. Phys. 93, 5978 (2003).

${ }^{7}$ H. W. Choi, C. W. Jeon, and M. D. Dawson, IEEE Photonics Technol. Lett. 15, 510 (2003).

${ }^{8}$ E. Gu, H. W. Choi, G. McConnell, C. Liu, C. Griffin, J. M. Girkin, I. M. Watson, and M. D. Dawson, Appl. Phys. Lett. 84, 2253 (2004). 